\title{
Comentarios a 'Aplicación de un modelo de trazadores lagrangianos en Bahía Anegada, Argentina' de Alvarez et al. (2011)
}

\author{
Comments on 'Aplicación de un modelo de trazadores lagrangianos en Bahía Anegada, \\ Argentina' by Alvarez et al. (2011)
}

Héctor H. Sepúlveda ${ }^{1}$ y Gonzalo Olivares $^{2}$

\begin{abstract}
${ }^{1}$ Departamento de Geofísica, Universidad de Concepción, Avda. Esteban Iturra s/n, Barrio Universitario, Concepción, Chile ${ }^{2}$ Centro i-mar, Universidad de Los Lagos, Chinquihue km 6, Casilla 557, Puerto Montt, Chile·g.olivares@ulagos.cl
\end{abstract}

\begin{abstract}
The paper by Alvarez et al. (2011) describes the use of a hydrodynamic numerical model to study the dispersion and sedimentation of particles at 2 points in Bahía Anegada (Argentina). This study is aimed to study the role of Crassostrea gigas as an invasive species. It is concluded that the particles liberated at those points do not represent a risk to the adjacent littoral zone. We consider that the article lack an adequate presentation of objectives, methods, and over the entire hydrodynamic model is not well validated. These factors undermine substantially the conclusions obtained.
\end{abstract}

Key words: Numerical modeling, lagrangian tracers, validation

El artículo de Alvarez et al. (2011) 'Aplicación de un modelo de trazadores lagrangianos en Bahía Anegada, Argentina' presenta la aplicación de un modelo hidrodinámico numérico para estudiar la dispersión y sedimentación de partículas en 2 puntos de la Bahía Anegada (Argentina). En él se utiliza el modelo numérico MOHID para el cálculo de las corrientes hidrodinámicas de la zona, y su módulo lagrangiano para estudiar la dispersión de partículas que se pretende representen larvas de ostra Crassostrea gigas. El artículo concluye que las partículas liberadas en ambos puntos de Bahía Anegada permanecen en ella, sin representar un riesgo evidente de dispersión de esa especie al litoral adyacente. Si bien el enfoque metodológico que combina modelos hidrodinámicos y lagrangianos, así como la consideración del forzamiento atmosférico parece correcto, el artículo presenta varias carencias que, en nuestra opinión, debilitan fuertemente sus conclusiones.

Un artículo debiera exponer una hipótesis clara de trabajo y fijar objetivos que permitan someter a prueba esa hipótesis. El valor del trabajo, en tanto, se debiera juzgar por la coherencia entre los objetivos asociados a la hipótesis, los métodos que permiten alcanzar dichos objetivos, y la elaboración de potenciales predicciones que se desprendan de la hipótesis de trabajo. En el caso del artículo de Alvarez et al. (2011), la única hipótesis que el lector puede extraer de su introducción es que las larvas de Crassostrea gigas pueden ser exportadas por las corrientes fuera de Bahía Anegada y, por ello, constituir una amenaza para las comunidades autóctonas del resto del litoral. En ese contexto, los autores establecen como objetivo 'aplicar el modelo hidrodinámico MOHID, con su correspondiente módulo de trazadores lagrangianos, para caracterizar la circulación de agua en la región’. La frase anterior induce a error, pues señala como objetivo lo que en realidad es un método (la aplicación de un modelo hidrodinámico), oscureciendo de paso el objetivo que se persigue con ese método (caracterizar la circulación de agua en la región). La caracterización de la circulación hubiese podido ser entendida como objetivo si la hipótesis hubiera estado relacionada con la circulación. Sin embargo, ya se dijo que la hipótesis tiene que ver con el rol de la ostra como invasor del litoral exterior a Bahía Anegada. Los objetivos que, una vez alcanzados, hubieran permitido someter a prueba tal hipótesis, debieran haber estado relacionados con estimar la provisión de larvas a un dominio exterior de la Bahía y la aptitud de los sitios que reciben larvas para sostener una nueva población del invasor. Es claro que el objetivo explicitado en la introducción de poco ayuda a someter a prueba la hipótesis que, expresada en la misma introducción, se usa para justificar el trabajo. Un ejemplo de coherencia entre hipótesis, objetivos y métodos en el área disciplinaria en la que se inserta el artículo de Alvarez et al. (2011), se puede ver el artículo de Miyake et al. (2010). La hipótesis de trabajo mencionada en la introducción de ese artículo es que el suministro natural de larvas de abalón a un área de refugio en la bahía de Oshoro, Japón, es mucho mayor que aquel representado 
por las semillas artificiales de esa especie liberadas en el mismo refugio. Consecuentemente, los autores fijan como objetivo estudiar la distribución espacio-temporal de larvas en la Bahía, para lo cual implementan un modelo hidrodinámico acoplado a uno de seguimiento de partículas lagrangianas, con el cual simulan el movimiento de larvas en la zona de interés. El proceso de validación y el diseño experimental son bien descritos tanto en el texto como a través de figuras, lo que permite estimar el efecto de una zona refugio (sin extracción) en la disponibilidad de larvas en la bahía.

Una objeción mayor al trabajo de Alvarez et al. (2011) es el inadecuado nivel de validación presentado para el modelo hidrodinámico. La única mención respecto a la validación de las velocidades simuladas se realiza en el primer párrafo de la página 203. En él, se señala que las velocidades máximas simuladas se observaron en el canal de San Blas, alcanzando 1,84 $\mathrm{m} \mathrm{s}^{-1}$ durante el reflujo y 1,97 $\mathrm{m} \mathrm{s}^{-1}$ durante el flujo, y que esas velocidades concuerdan con las observaciones realizadas en ese lugar por Cuadrado \& Gómez (2010). No se muestran los valores de corriente medidos, ni tampoco se cuantifica las diferencias con las simulaciones efectuadas. De esa forma, aparte de declarar la concordancia, no se le entrega al lector ningún elemento que permita constatar tal afirmación, como podría ser un gráfico donde se muestre el ajuste de corrientes y marea. Por otra parte, en el párrafo que sigue, sin mostrar las series de tiempo simuladas y observadas, y sin un análisis cuantitativo de las mismas, se afirma que existe una correspondencia entre la serie de tiempo simulada de nivel del mar y la observada en la estación cercana, EMAC. Debido a que se usa esa comparación como base para validar el modelo hidrodinámico, nuevamente los autores declaran el modelo validado sin entregarle al lector fundamentos para ello. En este contexto, las futuras simulaciones que se indican en el último párrafo de la discusión, debieran presentar una mejor validación de la circulación en esta zona previo al uso de las corrientes calculadas en un modelo lagrangiano (e.g., Moreno-Navas et al. 2011). Por otro lado, se omite una presentación espacial detallada de la batimetría (e.g., en la fig. 1), en particular para el dominio anidado, así como una descripción de las condiciones de borde, y del proceso de estabilización, del cual se menciona que es de 20 días, sin ser claro si esto se aplica a los 2 dominios o solo al dominio analizado.

En referencia al diseño de los experimentos lagrangianos, los autores definen partículas que permanecen a la deriva por 20 días, lo que encuentra justificación en el tiempo de desarrollo de las larvas de ostra del Pacífico que se describe en literatura, sin aclarar su movilidad en la columna de agua durante la deriva o el asentamiento. Lo anterior se lee como una señal en el sentido de intentar trabajar sobre la hipótesis de la ostra como especie invasora más que sobre una hipótesis relacionada a la circulación en el área. Dada la marea semidiurna que domina el área de estudio, es esperable que el momento del ciclo mareal en el cual se produzca la liberación pueda tener alguna influencia sobre la dispersión de las partículas, efecto que podría ser evaluado cualitativamente al cabo de sólo un ciclo de mareas. En ese sentido, a menos que el forzamiento mareal experimente cambios significativos en una escala temporal mayor a 12 h, y aun cuando exista un flujo residual, no tiene sentido simular el comienzo de la liberación por más de un ciclo mareal. Los autores simulan 90 días de circulación basado en que el desove de la ostra ocurre durante los 3 meses de la temporada estival austral. Como el tiempo de deriva de las partículas simuladas no depende de la temperatura del agua, lo anterior lleva implícita la sospecha que tanto el viento climatológico impuesto al dominio de menor resolución, como el viento local impuesto al dominio de mayor resolución, son capaces de generar variabilidad del flujo a escala de días a semanas. Sin embargo, pese a tener 90 días de variabilidad de flujo, los autores decidieron restringir las simulaciones lagrangianas a la mitad de ese periodo, sin especificar las fechas para las que se simuló la deriva. Más aún, en atención a que es la variabilidad del viento el único factor que justificaría simular la liberación por más de 1 día, sorprende que no se clarifique el periodo sobre el cual se calcula los descriptores del forzamiento atmosférico local mostrados en la figura 3. La caracterización de la variabilidad temporal del forzamiento local hubiera resultado más útil para el diseño de los experimentos numéricos que estimadores de tendencia central como la magnitud media de la velocidad del viento. Además, hubiera sido útil contar con una descripción de la topografía del dominio de mayor resolución para evaluar que tan apropiado es extrapolar los datos de la estación EMAC a toda el área de simulación. Respecto al número de partículas liberadas en cada condición, hubiera sido deseable una mejor justificación de un número que parece excesivamente bajo, incluso para una descripción cualitativa de la dispersión (ver por ejemplo Brickman \& Smith 2002). En suma, la definición de los experimentos numéricos en base a cuyos resultados se pretende poner a prueba la hipótesis de Alvarez et al. (2011), resulta incompleta y en varios aspectos dificulta la comprensión de sus resultados.

Los 2 párrafos dedicados a los resultados de los experimentos lagrangianos, se apoyan exclusivamente en 
la figura 5. Debido a que en los métodos se señala que las simulaciones se extienden por 45 días y cada partícula dura 20 días, a falta de una explicación en la leyenda o el texto, al lector no le es posible saber si la figura muestra las partículas presentes en un instante determinado de las simulaciones, o si en ella se representa alguna clase de resumen para todas las partículas simuladas. Falta información sobre las características de las partículas (e.g., flotabilidad o comportamiento). La interpretación se vuelve más confusa cuando, inducido por la barra de colores (que no se menciona en la leyenda de la figura), se observa que las partículas a las que hace mención el texto no han alcanzado aún la edad adecuada para asentarse (i.e., 20 días). Si las partículas que se dispersan poco menos de $16 \mathrm{~km}$ lo hacen faltándoles aún 10 días de deriva, el lector se ve obligado a sospechar que existe la posibilidad de que al cabo de su vida larval sean transportadas a mayor distancia que la indicada por los autores. No se explica adecuadamente los parámetros asociados al proceso de sedimentación de las partículas, qué pasaría con ellas sobre la costa en bajamar, o los coeficientes de dispersión definidos para estas. Por otro lado, la simple comparación visual de la posición de las partículas (fig. 5) y el campo de corrientes (fig. 4, que carece de una escala de referencia para los vectores), lleva al lector a conclusiones muy distintas a las mencionadas por los autores. De acuerdo a las figuras, el eje de mayor variabilidad de la marea se orienta en dirección NW-SE, mientras el eje de mayor dispersión de partículas se orienta en dirección SW-NE. Dado lo anterior, no parece que las partículas que más se dispersan le deban su posición a la acción de la marea, sino a la del viento (fig. 3), cuyo eje de mayor variabilidad se orienta aproximadamente en la misma dirección que la de la máxima dispersión. No obstante, una exploración más acabada del punto anterior no es posible dada la escasa información respecto al forzamiento local del viento en el periodo que efectivamente fue simulada la dispersión; o dicho de otro modo, esa exploración no es posible debido a una pobre definición de los experimentos lagrangianos y a la falta de alineación de estos con los objetivos y la hipótesis del trabajo. La literatura ofrece un buen número de trabajos que se pueden tomar de ejemplo al momento de diseñar tales experimentos numéricos (e.g., Brochier et al. 2011, Hinrichsen et al. 2003, Lett et al. 2007), incluso en zonas cercanas (Campuzano et al. 2013).

\section{Agradecimientos}

Agradecemos las contribuciones de dos revisores anónimos, cuyos comentarios permitieron mejorar la presente nota.

\section{LITERATURA CITADA}

Alvarez LI, DG Cuadrado \& GME Perillo. 2011. Aplicación de un modelo de trazadores lagrangianos en Bahia Anegada, Argentina. Revista de Biología Marina y Oceanografía 46(2): 199-206.

Brickman D \& PC Smith. 2002. Lagrangian stochastic modeling in coastal oceanography. Journal of Atmospheric and Oceanic Technology 19: 83-99.

Brochier T, E Mason, M Moyano, A Berraho, F Colas, P Sangrà, S Hernández-León, P Ettahir \& C Lett. 2011. Ichthyoplankton transport from the African coast to the Canary Islands. Journal of Marine Systems 87(2): 109122.

Campuzano FJ, MD Mateus, PC Leitão, VH Marín, A Tironi, JO Pierini, AJP Sampaio, P Almeida \& RJ Neves. 2013. Integrated coastal zone management in South America: A look at three contrasting systems. Ocean \& Coastal Management 72: 22-35.

Coelho H, R Neves, M White, P Leitao \& A Santos. 2002. A model for ocean circulation on the Iberian coast. Journal of Marine Systems 32: 153-179.

Cuadrado DG \& EA Gomez. 2010. Geomorfología y dinámica del canal San Blas, provincia de Buenos Aires (Argentina). Latin American Journal of Sedimentology and Basin Analysis 17(1): 3-16.

Hinrichsen H-H, U Böttcher, FW Köster, A Lehmann \& MA St. John. 2003. Modelling the influences of atmospheric forcing conditions on Baltic cod early life stages: distribution and drift. Journal of Sea Research 49(3): 187201.

Miyake Y, S Kimura, T Kawamura, T Kitagawa, M Hara \& H Hoshikawa. 2010. Estimating larval supply of Ezo abalone Haliotis discus hannai in a small bay using a coupled particle-tracking and hydrodynamic model: insights into the establishment of harvest refugia. Fisheries Science 76(4): 561-570.

Moreno-Navas J, TC Telfer \& LG Ross. 2011. Application of 3D hydrodynamic and particle tracking models for better environmental management of finfish culture. Continental Shelf Research 31(6): 675-684. 